\title{
Adaptive Thresholding for Dark/Light States in Single Quantum Dot Blinking Fluorescence
}

\author{
A.L. Shchukina ${ }^{1,2}$ and I.Yu. Eremchev ${ }^{2}$ \\ ${ }^{1}$ Warsaw University, Centre of New Technologies, S. Banacha 2c, Warsaw, 02-097, Poland \\ ${ }^{2}$ Institute for Spectroscopy RAS, Fizicheskaya Str. 5, Moscow, Troitsk 142190, Russia
}

Keywords: quantum dots, semiconductors, blinking fluorescence, statistics, thresholding effects

Quantum dots (QDs) are semiconductor nanocrystals with fluorescent properties depending on their composition, size, shape, etc. Many types of QDs manifest so-called blinking fluorescence, also typical for the majority of single quantum emitters (organic molecules, dielectric nanoparticles, etc.) $[1,2]$. Blinking of QDs is observed during cw-laser excitation: intervals of various intensities alternate in QD fluorescence. These are «dark» and «light» intervals. Various theories and hypotheses on the nature of QD dark states pursue to explain the subject [3].
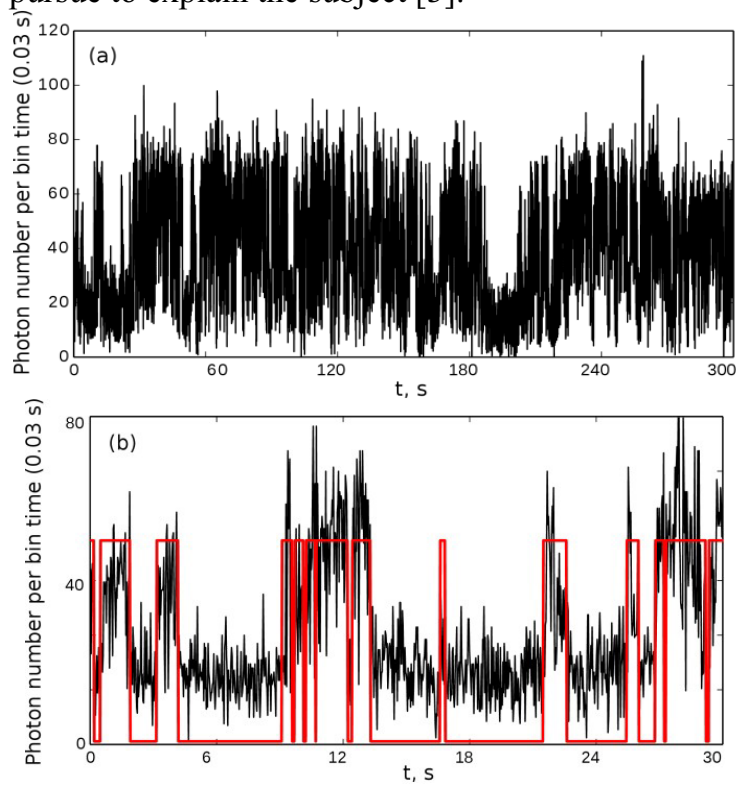

Figure 1. Fluorescence track of a single core-shell QD CdSe/ZnS. (a) Full track; (b) magnified selection. Grey line: the result of the suggested method of adaptive thresholding.

While analyzing the time track of single QD fluorescence it is necessary to distinguish between dark/light states intensities [1, 2, 4]. However, background fluorescence, detector noises, insufficient

This is an Open Access article distributed under the terms of the Creative Commons Attribution License 4.0, which permits unrestricted use, distribution, and reproduction in any medium, provided the original work is properly cited. 
temporary resolution of the experiment, etc. prevent from unambiguous determining of the QD states, which leads to an ambiguity of physical values extracted from the experimental data. A track of blinking fluorescence of a single core-shell QD CdSe/ZnS measured at room temperature [5], whose analysis is hampered by the difficulties listed above, is presented in Fig.1 (full track of 300s length in Fig.1a, its first 30s (magnified selection) in Fig.1b, black line).

To solve this problem, an original method was proposed in the present work. This method is based on the analysis of the fluorescence photon number distributions for QDs. For the simplest case of blinking fluorescence, such a distribution has two peaks: one at big photon numbers, corresponding to the light state of the emitter, and the other - at small numbers, corresponding to the dark state. Such a distribution for the same QD, data on which are given in Fig.1, is presented in Fig.2 with a solid black line. The approximation of the experimental peaks with normal distributions (Fig.2, dashed grey lines) enables to establish the borders, inside which this or that photon number refers (with a required probabilistic accuracy) to the light/dark state, and also to establish the interval of photon numbers, in which an exact conclusion on the state of a QD cannot be made. These borders are marked at the bottom of Fig.2.

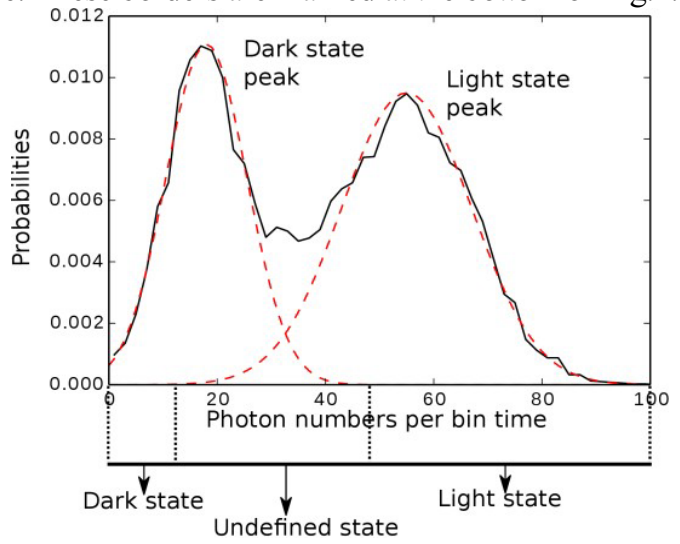

Figure 2. (Solid black line) Distribution of fluorescence photon numbers per bin time for blinking fluorescence of a single core-shell QD CdSe/ZnS. The two peaks correspond to the dark and the light state of the QD (Dashed grey lines) Approximation of the peaks by normal distributions. Under the abscise axis - intervals of photon numbers corresponding to: dark state; undefined interval; light state.

After the procedure illustrated in Fig.2, we return to the fluorescence track analysis. We determine the QD state at each measurement time point. The photon number at a given time point refers to one of three intervals: the dark state one, the light state one or the undefined one. In the latter case, neighboring time points (before and after the one under consideration) are taken into account. The results of the method are given in Fig. 1 b by grey line - light and dark intervals are identified. It appears that the suggested method can smoothen fluctuations within a dark/light state and identify it as one continuous light/dark, whereas thresholding with any fixed value cannot and would create «artifact» transitions, which do not really occur. To check this supposition, computer simulations will be carried out in our next works.

The authors would like to thank Dr. A.V. Naumov (Institute for Spectroscopy RAS, Moscow, Troitsk; Moscow State Pedagogical University) for fruitful discussions. The work has been supported by grant 14-02-31877 of Russian Foundation for Basic Research.

\section{References}

1. M. Kuno, D.P. Fromm, H.F. Hamann et al., J. Chem. Phys. 112, 3117 (2000)

2. S.V.Orlov, A.V.Naumov, Yu.G.Vainer, L.Kador, J. Chem. Phys. 137, 194903 (2012)

3. I.S.Osad'ko, I.Yu.Eremchev, A.V.Naumov, submitted in J. Phys. Chem. C (2015)

4. N. Amecke, A. Heber, F. Cichos, J. Chem. Phys. 140, 114306 (2014)

5. A. L. Shcukina, I. Yu. Eremchev, A. V. Naumov, Phys. Rev. E (2015) Article ID : LE15452E 